\title{
Extrusión húmeda del pasto Kikuyo (Cenchrus clandestinus (Hochst ex Chiov))
}

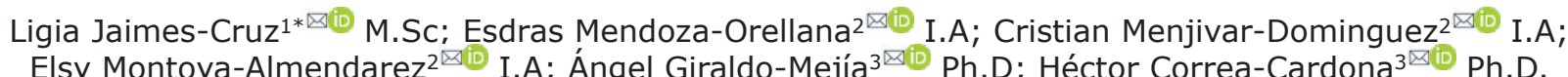

${ }^{1}$ Universidad Nacional de Colombia-Sede Medellín, Facultad de Ciencias Agrarias, Doctorado en Ciencias Agrarias; 2Universidad Nacional de Agricultura de Honduras;

3Universidad Nacional de Colombia-Sede Medellín, Facultad de Ciencias Agrarias, Departamento de Producción Animal.

*Correspondencia: ljjaimesc@unal.edu.co

Recibido: Marzo 2020; Aceptado: Octubre 2020; Publicado: Diciembre 2020.

\section{RESUMEN}

Objetivos. Evaluar el efecto de la extrusión húmeda sobre la digestibilidad in vitro (pepsina-celulasa) de la materia seca (DIVMS) y la fibra en detergente neutro (DIVFDN) del pasto kikuyo (Cenchrus clandestinus (Hochst ex Chiov)). Materiales y métodos. Se recolectaron seis muestras de esta gramínea cosechadas a los 35 días de rebrote $(5.0 \mathrm{~kg} /$ muestra), que fueron picadas a $2.0 \mathrm{~cm}$; de cada muestra se tomó una submuestra con la formación de una mezcla compuesta del pasto crudo en la que se determinó el contenido de proteína cruda (PC), fibra en detergente neutro (FDN), fibra en detergente ácido (FDA), lignina en detergente ácido (LDA), cenizas (Cen), calcio (Ca), fósforo $(P)$, potasio (K), la DIVMS y la DIVFDN; la cantidad restante de cada una de las seis muestra fue sometida la a extrusión húmeda en un extrusor de un solo tornillo cónico con salida de $2.0 \mathrm{~mm}$ que giraba a $1300 \mathrm{rpm}$. En los bagazos obtenidos de cada muestra se efectuaron los mismos análisis que en la muestra cruda. Los datos fueron analizados mediante una prueba de T de Student de dos colas. Resultados. Los bagazos obtenidos por extrusión húmeda del pasto kikuyo presentaron mayor contenido de FDN y FDA y menor de PC y $\mathrm{C}$ en en que el pasto crudo, al tiempo que se incrementó en más del $8 \%$ la DIVMS y en más del $36 \%$ de la DIVFDN. Conclusiones. La extrusión húmeda mejora significativamente la DIVMS y la DIVFDN del pasto kikuyo.

Palabras clave: Bagazo; biomasa; delignificación; digestibilidad; fibra; in vitro (Fuente: Thesaurus).

\section{ABSTRACT}

Objetives. Test the effect of wet extrusion on the in vitro dry matter (IVDMD) and neutral detergent fiber (IVNDFD) digestibility (pepsin-cellulase) of kikuyu grass (Cenchrus clandestinus (Hochst ex Chiov)). Materials and methods. Six samples of this pasture with 35 days of regrowth were collected $(5.0 \mathrm{~kg} / \mathrm{sample})$ and cut at $2.0 \mathrm{~cm}$; from each sample was took a sub-sample to create a composite sample of raw grass in which was analyzed the content of crude protein (CP), neutral detergent fiber (NDF), acid detergent fiber (ADF), acid detergent lignin (ADL), ashes, calcium (Ca), phosphorus $(P)$, potassium, IVDMD and IVNDFD; the rest quantity of each six samples was submitted to a wet 
extrusion in a single screw extruder machine with a $2.0 \mathrm{~mm}$ of output and $1300 \mathrm{rpm}$. In the bagasse obtained of each sample were determined the same analysis of the composite sample of raw grass. The data were analyzed by Student two tailed T-test. Results. The wet extrusion increased the fiber components and increased more of $8 \%$ the IVDMD and in more of $36 \%$ the IVNDFD of kikuyu grass. Conclusions. The wet extrusion in a technology that modified the chemical composition of the kikuyu grass and increase its digestibility.

Keywords: Bagasse; biomass; delignification; digestibility; in vitro (Source: thesaurus).

\section{INTRODUCCIÓN}

La producción de leche en las zonas frías de Colombia se realiza bajo pastoreo de diferentes tipos de gramíneas, donde el pasto kikuyo (Cenchrus clandestinus (Hochst ex Chiov)) es el más importante en los sistemas de lechería especializada en el departamento de Antioquia $(1,2,3)$. Es una gramínea C4 de origen sub-tropical que conserva las características anatómicas, fisiológicas y químicas de este tipo de plantas, lo que implica mayor acumulación de las paredes celulares y de la lignina que las gramíneas C3, lo que trae como consecuencia reducción en la digestibilidad de los carbohidratos estructurales $y$, por ende, reducción en la disponibilidad de la energía fermentable (4). Esto se acentúa mucho más si se tiene en cuenta que el contenido de carbohidratos no estructurales (CNE) en esta gramínea es bajo y el de proteína degradable (PDR) en rumen alto, generando desbalance entre estas fracciones que afecta, así mismo, la digestibilidad de la MS el rumen (1). Recientemente ha sido reportado que el valor nutricional de esta gramínea cambia poco con la edad de rebrote por lo que realizar el pastoreo a edades tempranas no incrementa la digestibilidad de la materia seca (5). Por ello esta gramínea no logra suplir la demanda de energía de las vacas cuyo nivel de producción supere los 11.5 litros/vaca/día $(6,3)$, en tal sentido, se precisa en estos casos, el uso de suplementos comerciales con alto contenido de almidón, lo que no solo reduce la digestibilidad de los carbohidratos estructurales $(7,8,9)$ sino que, incrementa los costos de producción (10), expone los animales a disfunciones alimenticias tales como las acidosis ruminales y las laminitis $(11,12)$ y puede generar un aumento en la producción de metano por animal/d debido a un aumento de la materia seca fermentada (13).

El efecto negativo que genera la lignina sobre la fermentabilidad de los carbohidratos estructurales de las paredes celulares (celulosa y hemicelulosa) constituye un limitante no solo en el ámbito de la nutrición de rumiantes, sino también, en la obtención de los combustibles de segunda generación (14) así como en la extracción de los derivados de los materiales lignocelulósicos que impliquen la fermentación o hidrólisis de la celulosa y la hemicelulosa (15). Para atenuar esta limitante, desde hace varias décadas se han desarrollado métodos químicos, biológicos y físicos conducentes a la reducción de los enlaces covalentes entre la lignina y estos carbohidratos estructurales (delignificación) (16). Entre los métodos físicos, la extrusión ha surgido como un pre-tratamiento rápido y de flujo continuo. Se trata de un proceso en el que el material vegetal es introducido a través de una camisa metálica cónica y es propulsado por un tornillo sin fin hacia el extremo más estrecho de la camisa, el cual termina en un dado con una salida cuyo tamaño se puede modificar. En la medida en que el material avanza por la camisa, se generan efectos de presión, temperatura y fuerza de cizalla sobre las paredes celulares del material procesado (17). Dicho proceso ha sido evaluado en materiales lignocelulósicos utilizados en producción de alcohol carburante siendo escasos los reportes sobre el uso de esta tecnología en forrajes utilizado en alimentación animal. Por ello, no existen reportes sobre el efecto de esta tecnología sobre la digestibilidad de las paredes celulares en gramíneas como el pasto kikuyo. Es por esta razón que el objetivo de este experimento fue evaluar el efecto de la extrusión húmeda del pasto kikuyo sobre la digestibilidad de la materia seca y de las paredes celulares.

\section{MATERIALES Y MÉTODOS}

Localización. Las muestras fueron recolectadas en la Estación Experimental Paysandú, ubicada en el corregimiento de Santa Elena (Medellín)

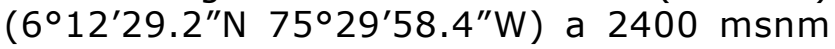
(bh-PM). 
Toma y procesamiento de muestras. Se recolectaron seis muestras de pasto kikuyo con 35 días de rebrote de un lote manejado de forma homogénea (aproximadamente $5.0 \mathrm{~kg} /$ muestra), las cuales fueron cortadas inmediatamente a $2.0 \mathrm{~cm}$. La edad de cosecha seleccionada corresponde a una de las más utilizadas en Antioquia (18).

Tratamientos experimentales. De cada una de las seis muestras se tomó una sub-muestra de $200 \mathrm{~g}$, las que se mezclaron y conformaron la muestra compuesta de pasto crudo. La cantidad restante de las seis muestras $(4.8 \mathrm{~kg} /$ muestra) fue sometida a la extrusión húmeda en un extrusor de un solo tornillo cónico con capacidad de procesamiento de aproximadamente $60 \mathrm{~kg}$ de materia verde/h (motor de $220 \mathrm{~V} ; 5.0 \mathrm{HP}$; $1300 \mathrm{rpm}$ ) y con salida de $2.0 \mathrm{~mm}$; del proceso de extrusión se retuvieron los bagazos.

Análisis químicos y físicos. En la muestra compuesta de pasto crudo y en los seis bagazos se determinó el contenido de proteína cruda $(\mathrm{PC})$, cenizas, calcio $(\mathrm{Ca})$, fósforo $(\mathrm{P})$ y potasio (K) (19), así como la fibra en detergente neutro (FDN), fibra en detergente ácido (FDA) y lignina en detergente ácido (LDA) (20). Se estimó el porcentaje de cada fracción química del pasto crudo que fue recuperada en los bagazos luego de la extrusión húmeda. Así mismo, todas las muestras se sometieron a una prueba de digestibilidad in vitro de la materia seca (DIVMS) y de la FDN (DIVFDN) mediante el método enzimático secuencial utilizando pepsinacelulasa (21).

Se llevó a cabo un análisis del tamaño de partícula para la muestra compuesta del pasto kikuyo crudo y para los bagazos obtenidos mediante la extrusión, para lo cual se utilizaron los tamices \# 4, 10, 14 y 20 (Pinzuar Ltda., Colombia), según procedimiento manual (22).

Análisis estadístico. Los datos obtenidos de los bagazos extruidos fueron sometidos al análisis estadístico frente a los resultados obtenidos de la muestra compuesta de pasto crudo mediante la prueba de T de Student de dos colas de control de calidad $(23,24)$.

\section{RESULTADOS}

En la Tabla 1 se muestra la composición química del pasto crudo y del bagazo. La composición química del bagazo mostró diferencia significativa con respecto al pasto crudo excepto en la LDA. El bagazo presentó contenidos más altos de FDN y FDA y más bajos en las demás fracciones.

Tabla 1. Comparación de la composición química del pasto kikuyo crudo y del bagazo obtenido por extrusión (\% de MS).

\begin{tabular}{ccccc}
\hline Fracción & Crudo & Bagazo & CME & p \\
\hline PC $^{*}$ & 14.9 & 12.8 & 0.403 & 0.001 \\
FDN & 68.6 & 77.3 & 1.54 & 0.001 \\
FDA & 34.7 & 38.0 & 0.14 & 0.001 \\
LDA & 4.67 & 5.22 & 0.382 & 0.154 \\
Cenizas & 10.1 & 5.56 & 0.065 & 0.001 \\
Ca & 0.415 & 0.310 & 0.0005 & 0.001 \\
P & 0.545 & 0.302 & 0.0002 & 0.001 \\
K & 2.67 & 1.05 & 0.012 & 0.001 \\
\hline
\end{tabular}

*PC: proteína cruda; FDN: fibra en detergente neutro; FDA: fibra en detergente ácido; LDA; lignina en detergente ácido; Ca: calcio; P: fósforo; K: potasio; CME: Cuadrado Medio del Error; p: probabilidad

La recuperación de las fracciones químicas en el bagazo fue variable (Tabla 2 ). Fue alta en las fracciones fibrosas (FDN, FDA y LDA), seguida de la PC y baja en las cenizas. Entre los minerales, sin embargo, hubo diferencias resultando más alta la recuperación en el Ca y menor en el $\mathrm{P}$ y K.

Tabla 2. Recuperación de las fracciones químicas del pasto kikuyo crudo en el bagazo obtenido mediante extrusión.

\begin{tabular}{ccc}
\hline \multirow{2}{*}{ Fracción } & \multicolumn{2}{c}{ Recuperación, \% } \\
\cline { 2 - 3 } & Promedio & DE \\
\hline PC & 74.0 & 2.73 \\
FDN & 97.1 & 2.02 \\
FDA & 94.5 & 3.35 \\
LDA & 97.0 & 17.0 \\
Cenizas & 47.4 & 1.25 \\
Ca & 64.4 & 2.32 \\
P & 47.7 & 1.75 \\
K & 33.9 & 1.94 \\
\hline
\end{tabular}

*PC: proteína cruda; FDN: fibra en detergente neutro; FDA: fibra en detergente ácido; LDA; lignina en detergente ácido; Ca: calcio; P: fósforo; K: potasio; DE: desviación estándar

El efecto de la extrusión húmeda del pasto kikuyo sobre la DIVMS y la DIVFDN se presenta en la Tabla 3. Se observó que, el proceso de extrusión incrementó la DIVMS en cerca del $8 \%$ 
y la DIVFDN en más del $36 \%$ comparado con el pasto crudo, lo que sugiere un efecto positivo del proceso de extrusión sobre la digestibilidad de los componentes de la pared celular.

Tabla 3. Digestibilidad in vitro de la materia seca (DIVMS) y de la fibra en detergente neutro (DIVFDN) del pasto kikuyo crudo y extruido.

\begin{tabular}{ccccc}
\hline Fracción & Crudo & Bagazo & CME $^{*}$ & p \\
\hline DIVMS & 41.0 & 42.2 & 2.44 & 0.005 \\
DIVFDN & 29.3 & 39.9 & 3.54 & 0.001 \\
\hline
\end{tabular}

${ }^{*}$ CME: Cuadrado Medio del Error; p: probabilidad

En la Tabla 4 se presenta la distribución del tamaño de partícula del pasto kikuyo crudo y cortado, así como del extruido. Como se puede apreciar el proceso de extrusión no modificó el porcentaje de partículas retenidas en los tamices número 4 y 10 , pero redujo la proporción de partículas en los tamices número14 y 20.

Tabla 4. Distribución del tamaño de partícula del pasto kikuyo crudo y del bagazo extruido.

\begin{tabular}{ccccc}
\hline Tamiz \# & Crudo & Bagazo & DE$^{*}$ & p \\
\hline $4(4.75 \mathrm{~mm})$ & 58.5 & 58.2 & 2.22 & 0.797 \\
$10(2.0 \mathrm{~mm})$ & 28.5 & 30.6 & 1.78 & 0.175 \\
$14(1.4 \mathrm{~mm})$ & 2.81 & 0.48 & 0.049 & 0.000 \\
$20(850 \mathrm{~mm})$ & 5.15 & 2.01 & 0.259 & 0.002 \\
Fondo & 4.99 & 8.72 & 2.24 & 0.102 \\
\hline
\end{tabular}

${ }^{*}$ DE: Desviación estándar; p: probabilidad

\section{DISCUSION}

Debido a que la extrusión húmeda es un proceso que separa el contenido celular del de las paredes celulares, se esperaba cambios en la composición química del bagazo frente al pasto crudo. Aunque el contenido de PC se redujo en el bagazo, este sin embargo continuó siendo importante desde el punto de vista nutricional y suficiente para soportar un nivel de producción de leche de aproximadamente $14.0 \mathrm{l} / \mathrm{d}$ en una vaca de $550 \mathrm{~kg}$ de peso vivo (25). Esto significó que el bagazo, no obstante, de haber presentado una composición química diferente a la del pasto crudo, su potencial de producción de leche continuó siendo importante con la ventaja que permite su conservación como tal o ser peletizado, lo cual facilita su almacenamiento y manejo.
La recuperación de las diferentes fracciones químicas en el bagazo depende de su ubicación entre el contenido o las paredes celulares. Como ha sido observado con el bagazo de la caña de azúcar (26), las fracciones químicas asociadas a las paredes celulares (FDN, FDA y LDA) fueron las que más se recuperaron en el bagazo del pasto kikuyo (Tabla 2). Entre los minerales, se observó que la recuperación del Ca fue mayor debido, posiblemente, a que este se encuentra estrechamente ligado a las paredes celulares $(27,28)$ mientras que el $\mathrm{K}$ presentó el menor porcentaje de recuperación en el bagazo debido a que se trata de un ion de alta solubilidad en la fracción líquida de los tejidos vegetales (27).

El proceso de extrusión mejoró la DIVMD y la DIVFDN (Tabla 3 ). Ha sido reportado que la extrusión semi-seca genera un aumento significativo en la digestibilidad de DIVMS y DIVFDN del pasto Pheleum pratense L., debido probablemente a que dicho proceso afecta la estructura física y química de esta gramínea (29). Otros autores han encontrado, así mismo, aumentos en la fermentabilidad de otras gramíneas forrajeras luego de su procesamiento mediante diferentes tipos de extrusores, expresada está en el incremento en el rendimiento de diferentes monosacáridos producto de la degradación de la celulosa y hemicelulosa (glucosa, xilosa, arabinosa) $(30,31)$.

La proporción de partículas retenidas en los tamices números 4 y 10 del bagazo extruido fue superior al $88 \%$ y superan el mínimo recomendado de $1.18 \mathrm{~mm}$ (32). Esto sugiere que el consumo de cantidades significativas de bagazo de pasto kikuyo obtenido por extrusión de acuerdo con el procedimiento descrito aquí, no se asociaría con riesgos de acidosis ruminal. Este efecto, sin embargo, requiere ser evaluado in vivo para la verificación de la relación de la distribución del tamaño de partícula en el bagazo de pasto kikuyo con el $\mathrm{pH}$ ruminal.

En conclusión, el proceso de extrusión experimentado permitió la obtención del bagazo del pasto kikuyo cuyo contenido de fracciones fibrosas resultó mayor que en el pasto crudo, con la retención de una porción importante de la PC con un alto potencial para ser utilizado en la suplementación de rumiantes. Este proceso aumento en más del $8 \%$ la DIVMS y en más del $36 \%$ la DIVFDN, con una reducción de la necesidad de utilizar suplementos ricos en almidones en la alimentación de rumiantes al mejorar el aporte de energía fermentable en el rumen. 


\section{Conflicto de interés}

Los autores de este manuscrito manifestamos que la publicación de este estudio no genera conflicto con nuestras actividades profesionales.

\section{Agradecimientos}

Este trabajo fue financiado por el proyecto 44321 de la convocatoria para el Apoyo al Desarrollo de Tesis de Posgrado de la Universidad Nacional de Colombia 2018, sede Medellín.

\section{REFERENCIAS}

1. Correa HJ, Pabón ML, Carulla JE. Valor nutricional del pasto kikuyo (Pennisetum clandestinum Hoechst Ex Chiov.) para la producción de leche en Colombia (Una revisión): I - Composición química y digestibilidad ruminal y posruminal. Liv Res Rural Dev. 2008; 20:Article59. http://www. Irrd.org/Irrd20/4/corra20059.htm

2. Correa HJ, Rodríguez YG, Pabón $M L$, Carulla JE. Efecto de la oferta de pasto kikuyo (Pennisetum clandestinum) sobre la producción, la calidad de la leche y el balance de nitrógeno en vacas Holstein. Liv Res Rural Dev. 2012; 24:Article204. http:// www.Irrd.org/Irrd24/11/corr24204.htm

3. Jaimes LJ, Correa HJ. Balance de nitrógeno, fósforo y potasio en vacas Holstein pastando praderas de kikuyo (Cenchrus clandestinus) en el norte de Antioquia. Rev. CES Med. Zootec. 2016; 11(2):18-41. http:// revistas.ces.edu.co/index.php/mvz/article/ view/3959/2629

4. Yahaya M, Goto M, Yimiti W, Smerjai B, Kawamoto Y. Evaluation of Fermentation Quality of a Tropical and Temperate Forage Crops Ensiled with Additives of Fermented Juice of Epiphytic Lactic Acid Bacteria (FJLB). Asian-Australas J Anim Sci. 2004; 17(7):942-946. https://doi.org/10.5713/ ajas.2004.942

5. Correa HJ, Escalante LF, Jaimes LJ.: Efecto de la época del año y la altura remanente posterior al pastoreo sobre el crecimiento y calidad nutricional del pasto kikuyo (Cenchrus clandestinus) en el norte de Antioquia. Liv Res Rural Dev. 2018; 30:Article97 http://www.Irrd.org//rrd30/6/ hjcor30097.html
6. Correa HJ, Pabón ML, Carulla JE. Valor nutricional del pasto kikuyo (Pennisetum clandestinum Hoechst Ex Chiov.) para la producción de leche en Colombia (Una revisión): II - Contenido de energía, consumo, producción y eficiencia nutricional. Liv Res Rural Dev. 2008b; 20:Article61. http://www. Irrd.org/Irrd20/4/corr20061.htm

7. Luo G, Xu W, Yang J, Li Y, Zhang L, Wang $Y$, Lin C, Zhang $Y$. Effects of ruminally degradable starch levels on performance, nitrogen balance, and nutrient digestibility in dairy cows fed low corn-based starch diets. Asian-Australas J Anim Sci. 2017; 30(5):653-659. https://doi.org/10.5713/ ajas. 16.0371

8. de Souza RA, Tempelman RJ, Allen MS, Weiss WP, Bernard JK, VandeHaar MJ. Predicting nutrient digestibility in high-producing dairy cows. J Dairy Sci. 2018; 101:1123-1135. https://doi.org/10.3168/jds.2017-13344

9. Quang DV, Nguyen XB, Peter TD, Dau VH, Peter AL, Aduli MA, Nguyen HV, David P. Effect of concentrate supplementation on nutrient digestibility and growth of Brahman crossbred cattle fed a basal diet of grass and rice Straw. J Anim Sci Tech. 2015; 57:35. https://doi.org/10.1186/s40781015-0068-y

10. Machado LC, D'Ávila LM, da Silva DC, Bento LL, Kuhnen S. Productive and Economic Responses in Grazing Dairy Cows to Grain Supplementation on Family Farms in the South of Brazil. Animals (Basel). 2014; 4(3):463-475. https://doi.org/10.3390/ ani 4030463 
11. Wang $H$, Pan $X$, Wang $C$, Wang $M, Y u L$. Effects of different dietary concentrate to forage ratio and thiamine supplementation on the rumen fermentation and ruminal bacterial community in dairy cows. Anim Prod Sci. 2015; 55(2):189-193. https:// doi.org/10.1071/AN14523

12. Abdela, N. Sub-acute Ruminal Acidosis (SARA) and itConsequence in Dairy Cattle: A Review of Past and Recent Research at Global Prospective. Achiev Life Sci. 2016; 10(2):187-196. https://doi.org/10.1016/j. als.2016.11.006

13. Na Y, Li DH, Lee SR. Effects of dietary forageto-concentrate ratio on nutrient digestibility and enteric methane production in growing goats (Capra hircus hircus) and Sika deer (Cervus nippon hortulorum). Asian-Aust J Anim Sci. 2017; 30(7):967-972. https:// doi.org/10.5713/ajas.16.0954

14. Robak K, Balcerek M. Review of secondgeneration bioethanol production from residual biomass. Food Tech Biotech. 2018; 56(2):174-187. https://doi.org/10.17113/ ftb.56.02.18.5428

15. Kucharska K, Rybarczyk P, Hołowacz I, Łukajtis R, Glinka M, Kamiński M. Pretreatment of lignocellulosic materials as substrates for fermentation processes. Molecules. 2018; 23(11):2937. https://doi. org/10.3390/molecules23112937

16. Agbor VB, Cicek N, Sparling R, Berlin A, Levin DB. Biomass pretreatment: Fundamentals toward application. Biotech Adv. 2011; 29:675-685. https://doi.org/10.1016/j. biotechadv.2011.05.005

17. Lin Z, Liu L, Li R, Shi J. Screw extrusion pretreatments to enhance the hydrolysis of lignocellulosic biomass. J Micr Bioch Tech. 2012; 5(S12):5. https://doi. org/10.4172/1948-5948.S12-002

18. Arango J, Cardona FA, López A, Correa G, Echeverri JJ. Variación de caracteres morfológicos del pasto kikuyo (Cenchrus clandestinus) en el trópico alto de Antioquia. Rev. CES Med. Zootec. 2017; 12(1):44-52. https://dx.doi.org/10.21615/cesmvz.12.1.4
19. Association of Official Analytical ChemistAOAC. Methods of Analysis. 20 ed. Washington D.C.: AOAC Int.; 2016.

20. Van Soest PJ, Robertson JB, Lewis BA. Methods for dietary fibre, neutral detergent fibre, and non-starch polysaccharides in relation to animal nutrition. J Dairy Sci. 1991; 74:3583-3597. https://doi. org/10.3168/jds.S0022-0302(91)78551-2

21. Barchiesi C, Alomar D, Miranda H. PepsinCellulase Digestibility of Pasture Silages: Effects of Pasture Type, Maturity Stage, and Variations in the Enzymatic Method. Chilean J Agric Res. 2011; 71(2):249257. https://doi.org/10.4067/S0718$\underline{5839201100020001}$

22. Tayyab U, Wilkinson RG, Reynolds CK, Sinclair LA. Particle size distribution of forages and mixed rations, and their relationship with ration variability and performance of UK dairy herds. Liv Sci. 2018; 217:108-115. https://doi.org/10.1016/j. livsci.2018.09.018

23. Gerald, B. A Brief Review of Independent, Dependent and One Sample t-test. Int J Appl Math Theor Phys. 2017; 4(2):50-54. https:// doi.org/10.11648/j.ijamtp.20180402.13

24. Feng $Y$, Huang $Y, M a X$. The application of Student'st-test in internal quality control of clinical laboratory. Front Lab Med. 2017; $1: 125-128$. https://doi.org/10.1016/j. flm.2017.09.002

25. Tedeschi LO, Fox DG, Fonseca MA, Cavalcanti LF. Models of protein and amino acid requirements for cattle. $\mathrm{R}$ Bras Zootec. 2015; 44(3):109-132. https://doi.org/10.1590/ $\underline{\text { S1806-92902015000300005 }}$

26. Ribeiro AF, Messana JD, Neto AJ, Lage JF, Fiorentini G, Vieira BR, Berchielli TT. Enteric methane emissions, intake, and performance of young Nellore bulls fed different sources of forage in concentraterich diets containing crude glycerine. Anim Prod Sci. 2016; 58:517-522. https://doi. org/10.1071/AN15645 
27. Fry S. Primary cell wall metabolism: tracking the careers of wall polymers in living plant cells. New Phyt. 2004; 161:641675. https://doi.org/10.1111/j.14698137.2004.00980.x

28. Thor K. Calcium-Nutrient and Messenger. Front Plant Sci. 2019; 10:440. https://doi. org/10.3389/fpls.2019.00440

29. Elgemark E. Intensively processed silage using Bio-extruder. Swedish University of Agricultural Sciences, Department of Animal Nutrition and Management: Uppsala; 2019. https://stud.epsilon.slu.se/14511/7/ Elgemark E 190405.pdf

30. Khor WC, Vervaeren H, Rabaey K. Combined extrusion and alkali pretreatment improves grass storage towards fermentation and anaerobic digestión. Biom Bioen. 2018; 119:121-127. https://doi.org/10.1016/j. biombioe.2018.09.003
31. Jurišić $\mathrm{V}$, Julson JL, Krička $\mathrm{T}$, Ćurić $\mathrm{D}$, Voća N, Karunanithy C. Effect of extrusion pretreatment on enzymatic hydrolysis of miscanthus for the purpose of ethanol production. J Agr Sci. 2015; 7(11):132-142. https://doi.org/10.5539/jas.v7n11p132

32. Maulfair DD, Fustini M, Heinrichs AJ. Effect of varying total mixed ration particle size on rumen digesta and fecal particle size and digestibility in lactating dairy cows. J Dairy Sci. 2011; 94:3527-3536. https:// doi.org/10.3168/jds.2010-3718 